\title{
A Study of Non-linear Dynamic Aerodynamic Behaviour of a Specialised Delta Wing
}

\author{
Christopher Pevitt ${ }^{1} \&$ Firoz Alam ${ }^{1}$ \\ ${ }^{1}$ School of Aerospace, Mechanical and Manufacturing Engineering, RMIT University, Melbourne, Australia \\ Correspondence: Christopher Pevitt, School of Aerospace, Mechanical and Manufacturing Engineering, RMIT \\ University, Melbourne, Australia. Tel: 61-423-031-275. E-mail: christopher.pevitt@student.rmit.edu.au
}

Received: March 29, 2012

Accepted: May 4, 2012 Online Published: May 14, 2012

doi:10.5539/mas.v6n6p18

URL: http://dx.doi.org/10.5539/mas.v6n6p18

\begin{abstract}
The objective of this paper was to model the stability and control derivatives using Computational Fluid Dynamics (CFD). This would provide a more reliable tool in the development of aircraft. This process could reduce the reliance on wind tunnel results with a consequent reduction in development costs. The test model used for this paper was a specialised delta wing configuration. The study was undertaken by comparing simulation parameters and determining their effects on the flow characteristics. The simulation was undertaken using internal meshing software, the flow simulation software TAU and the graphical interface Tecplot. Results showed that a single CFD model could not be used for the prediction of aerodynamic behaviour under the full range of angle of attack $\left(0^{\circ}\right.$ to $\left.25^{\circ}\right)$. However the surface mesh refinement and optimisation of simulation parameters allowed for a better prediction at lower angle of attack $\left(0^{\circ}\right.$ to $\left.15^{\circ}\right)$. The dynamic simulation showed that flow characteristics were better captured for higher pitching frequencies. Overall the study will assist the progress of future studies.
\end{abstract}

Keywords: computational fluid dynamics modelling, delta wing, stability and control, dynamic behaviour, vortex, flow separation

\section{Introduction}

Computational Fluid Dynamics (CFD) modelling has been used to a significant extent in the development of aircraft. As capabilities and computing power, of CFD modelling, have increased over time, the importance of and reliance upon, CFD simulations has similarly increased. Simulation results, obtain over time have progressively become more accurate and reliable. However the data provided by CFD simulations has had limitations. As such, the results of CFD simulations have never been relied on, as the sole source of data. The results were always validated with additional data obtained, either via wind tunnel or flight tests, both of which are costly and time consuming. Also, these options are not always practical or suitable alternatives. Additionally, under certain flight characteristics the wind tunnel results do not entirely represent the true flow over an aircraft.

It is preferable if all flight characteristics are known before the full scale aircraft enters the flight testing. Once full scale flight testing commences, it is costly and time consuming to modify the design. Furthermore, unexpected aircraft handling during testing can be very dangerous. Therefore, it would be greatly beneficial to improve the reliability and accuracy of current CFD modelling, which would reduce the necessity for additional alternative testing. This would help to reduce costs, in addition to opening up possibilities for more detailed testing under the entire flight envelope of the aircraft.

With recent advances in the aerospace industry, the demand and commonality of Unmanned Aerial Vehicles (UAV) have also increased. The leading factor in this area is Unmanned Combat Aerial Vehicles (UCAV). These planforms often lead to configuration with nonlinear aerodynamic behaviour; this can be dominated by vortical flow across the upper surfaces. The cause of these more complicated flows is often linked to UCAV's highly swept wing planform design. Many of the characteristics of flow phenomena associated with highly swept delta wings have been well documented and well-studied (Gursul, Gordinier, \& Visbal, 2005). These planforms often are associated with the sharp leading edge geometry. However, the flow is not entirely understood in less swept wings with rounded leading edges. It is these characteristics that need to be better understood in order to effectively enhance future development of aircraft. 
The determination of dynamic derivatives in aircraft development is an essential part of the development cycle in flight physics. When reviewing an unstable aircraft, knowledge of the stability and control margins are critical for the design of flight control systems. This is vital, as many future unmanned aircraft configurations exhibit aerodynamic stability and control issues in various regions of their flight envelopes.

The primary objective of this study is to focus on the computation of the pitch oscillations in the interesting angle of attack (AoA) range $\left(0^{\circ}\right.$ to $\left.15^{\circ}\right)$. During this process individual parameters will be assessed. These include the dependencies on configurations (with and without sting), mesh resolutions, discretisation schemes, turbulence and transition models, time step sizes and order of the time integration operations. The results of these tests will then be compared to the characteristics of flow found in the wind tunnel testing.

\section{Delta Wing Configurations}

As technology and demands on aircrafts are advancing and increasing, the desire for additional speeds and manoeuvrability capabilities are becoming more necessary. These factors should be considered in the design and developmental phase of modern aircraft. The configurations associated with both supersonic and subsonic aircrafts vary greatly. The delta wing is not new technology; as its initial concept was developed in 1867 (Century of Flight, 2010). It is one of the most efficient ways to achieve the desired high speed capabilities of a wing. Delta wings are a common feature for aircrafts tailored for the supersonic flight. The majority of modern aircraft have some aspects of swept wings to gain the beneficial effect of preventing high speed shock effects (Gursul, 2005). There are a large number of delta wing types including; Standard, Ogival, Compound, Cropped, Tailless, Cranked Arrow and Diamond/Lambda configurations (Surruno, 2009) as shown in Figure 1.

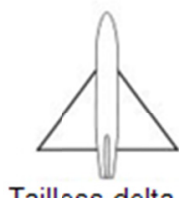

Tailless delta

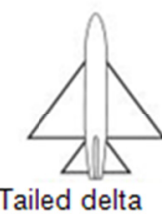

Tailed delta

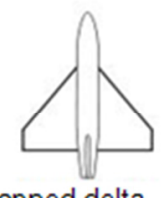

Cropped delta

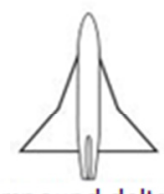

Compound delta

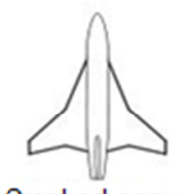

Cranked arrow

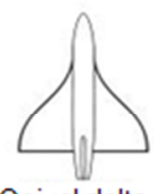

Ogival delta

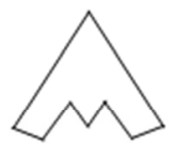

Lambda delta

Figure 1. Delta Wing Configurations (Surruno, 2009)

In this study a Lambda type delta wing with a $53^{\circ}$ swept wing was used (Visbal, 1995). The selected Lambda delta wing possesses a low sweep angle and non-slender characteristic (Gursul, 2005).

Additionally it has a combination of both rounded and sharp leading edge geometry. The model is a specifically designed UCAV delta wing configuration. It was designed in order to develop key aerodynamic characteristics such as flow separation and the development of vortices (Anderson, 2007). The exact configuration used cannot be shown here due to current confidentially restrictions. The model has a $52^{\circ}$ swept leading edge with the capability of interchanging a sharp or rounded leading edge. For this study, the rounded leading edge would be used. The rounded leading edge configuration is created with a sharp inboard leading edge which transitions into a medium round leading edge on the outer panels of the wing. The outer panel has a parallel leading and trailing edge with a washout twist of $5^{\circ}$ (Frink, 2010).

The model consists of three main sections; the fuselage, the wing and wing tips. It is made of a light weight reinforced plastic that brings its overall weight to less than $10 \mathrm{~kg}$ (Vicroy, Loeser, \& Schütte, 2010). The purpose of the extra light model is that it reduces the dynamic inertial loads (Yang X. L. et al., 2011). This allows for a more accurate and sensitive balance, which leads to better force and moment resolution. The model consists of more than 200 pressure taps on the upper and lower sides of the model which are set to determine the dynamic measurements of unsteady pressure. The model was designed to gather both static and dynamic aerodynamic behaviour. However for this study, the results are restricted to dynamic behaviour modelling.

\section{The DLR Tau-code}

The Flow simulation software used for this study was the DLR TAU-Code. This is a software modelling package developed by the DLR Institute of Aerodynamics and Flow Technology. It was designed to be capable of solving complex CFD simulations. The solver is based on the compressible three-dimensional, steady, and unsteady Reynolds Averaged Navier-Stokes equations (Shütte, 2010). This is implemented by employing finite volume flow solutions. The code is established from a hybrid, unstructured grid approach. This allows implementing multiple forms of grids, leading to the capability of analysing much more complicated and detailed models (Kollmann, 2011). The unstructured grid consists of both hexahedral and prismatic grids. This offers better resolution within the viscous shear layers, to accurately represent near wall conditions, through the use of a 
structured prismatic grid (Shütte, 2010). Additionally, it allows for the utilisation of the freedom and capabilities of the unstructured volume mesh. In this study, an unstructured grid was used that is developed with an in-house meshing program called "Mesher".

The TAU-Code has the capabilities to utilise both the Cell-Vertex and the Cell-Centered schemes, both with their own advantages and disadvantages (Liu \& Chen, 2011). Here the Cell-Centered scheme was used. In the Cell-Centered approach, the Navier-Stokes equations are solved on a dual background grid, which is determined directly from the primary grid (Hübner, 2007). This approach was used as it consists of a larger number of solution variables than other approaches, which would in turn lead to greater accuracy.

The TAU-Code is capable of performing many different tasks splitting into five main modules. These modules are (Shütte, 2010): a) Preprocessor - Takes information from the Primary grid to develop a dual-grid or multi-grids. b) Solver - Performs the flow calculations over the dual-grid. c) Adaption - Refines and de-refines the grid to allow for the capture of all flow phenomena. This includes a large range of categories, including the representation of vortex structures and shear layers around viscous boundaries. d) Deformation - Propagates the deformation of surface-coordinates to the surround grid. e) Motion - Defines the motion of the model and relates this motion to any control devices. Many of these modules are inbuilt within the code and for this study; they would not be altered from their default values. As a result, the Preprocessor and Solver modules would be examined in more details while the other modules would be taken as non-variable.

The Preprocessor module is based on the meshed grid forming the primary grid. Here, a system of five dual grids was used. This introduction of multiple grids greatly improves the computational time and power required to run any simulation.

The Solver module calculates the gradients in time, which are then discretised through the use of a multi-step Runge-Kutta scheme. These calculations are then calculated using multigrid techniques and local time stepping which accelerates the ability to find converged results for steady state solutions (Hübner, 2007). Three different turbulence models were examined: a) one one-equation model, b) two two-equation models. These were the Spalart-Allmaras Edwards one-equation model, and the Wilcox k- $\omega$ TNT and Wilcox k- $\omega$ LEA two-equation models

\section{Numerical Results}

\subsection{Findings of Initial CFD Modelling}

In order to determine the possible accuracy of CFD simulations and how different parameters can be improved, the quality of the initial simulations results are needed. To be able to check these parameters accurately it is important to ensure a good quality grid density (Liu \& Chen, 2011). The mesh can greatly affect the results of the simulation. As a consequence it is vital to ensure that any simulations being used have reached a mesh converged state (Ismadi, 2011). For this work a large array of simulations was developed with meshed densities ranging from 1.5 to 22.5 million nodes. These simulation results were compared to wind tunnel findings to determine the accuracy of each model. A detailed look at this mesh convergence study was undertaken in a previous study by Pevitt \& Alam (2011).

The wind tunnel investigations were undertaken across two wind tunnel facilities with a total of three full scale investigations. The first two of these tests were conducted in DNW-NWB wind tunnel and the final test in NASA Langley 14-by-22-Foot Subsonic Tunnel in Hampton, Virginia (Vicroy, 2010). For this work the results obtained from the DNW-NWB wind tunnel will be used. Dynamic measurements of integral forces and moments, the pressure distribution over the wing surface, transition measurements and field measurements (both static and dynamic) using Particle Image Velocimetry (PIV) were taken (Shütte, 2010) .

The DNW-NWB wind tunnel is a closed loop, with an atmospheric test section, capable of operating under both open slotted or closed configuration (Cummings et al., 2010). The wind tunnel is $3.25 \mathrm{~m}$ by $2.8 \mathrm{~m}$ and has a maximum free stream velocity of $80 \mathrm{~m} / \mathrm{s}$ for the closed test section and $70 \mathrm{~m} / \mathrm{s}$ for the open test section (Vicroy, 2010). The wind tunnel data collected at a speed of $50 \mathrm{~m} / \mathrm{s}$ will be used for this study, corresponding to a Reynolds number of 1.57 million and a Mach number of 0.147 (Cummings, 2010). The model was tested statically at an angle of attack range of $-15^{\circ}$ to $30^{\circ}$ and dynamically under pitch and yaw with oscillation of $\pm 5^{\circ}$ amplitude (Vicroy, 2010). For the work being done the pitch oscillation results will be used for angles of attack between $0^{\circ}$ and $25^{\circ}$.

The previous mesh convergence study found that there were considerable differences in changing meshes. When reviewing the $C_{L}$ (Coefficient of Lift) and $C_{D}$ (Coefficient of Drag) values all of the meshes appeared quite capable of representing the experimental flow characteristics. While the $\mathrm{C}_{\mathrm{my}}$ (Coefficient of Pitching Moment) 
values were much more unstable and were proven to be highly mesh dependent (Pevitt \& Alam, 2011). To further understand why there is a difference in the results of each of the meshes, the surface contours were taken of the pressure coefficient over the configuration, which help to visualise the vorticity formation over the surface of the wing. These comparisons are shown for an AoA of $15^{\circ}$ in Figure 2.

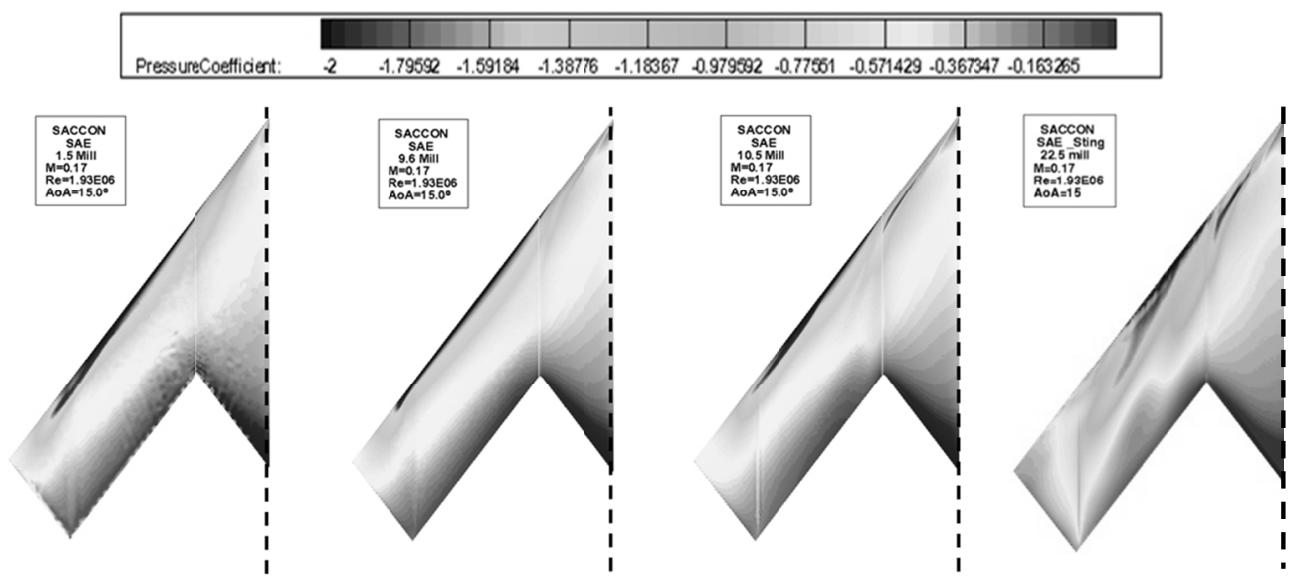

Figure 2. Surface Contour Plots at $15^{\circ}$ AoA, Comparing Mesh Refinement (Pevitt \& Alam, 2011)

The pressure contours shown in Figure 2 indicated critical differences in the flow characteristics for each of the meshes. Here four meshes were depicted as they showed the range from undefined to refined meshes. With additional meshes closer to the mesh convergence point displayed, initially seen at 10.5 million nodes. This was deemed a critical point in the mesh convergence study, as the complex flow over the surface of the wing indicated early signs of mesh convergence here, though it was not until further testing and comparisons to wind tunnel results were made that it was found that the mesh did in fact not converge until a mesh density of 22.5 million nodes was reached. Experimental findings indicated that at $15^{\circ}$ AoA there should be the beginning of a dual vortex formation occurring, though this was not observed in CFD simulations until addition mesh refinements were made (Pevitt \& Alam, 2011), (Kuo et al., 1995). The final mesh ended up with the best representation of the flow as well as satisfying critical mesh features like a low $\mathrm{Y}+$ values, which was noted at 1 for the 22.5 million node model (Pevitt \& Alam, 2011). This resulted in a smoother, more accurate mesh, better capable of accurately modelling the flow characteristics over the configuration (Zhang, 2011).

\subsection{Sensitivity of Turbulence Models}

When analysing the flow over the configuration, it is vital to determine the sensitivity of the results due to the turbulence models (Kwak, 2010). All turbulence models are developed for different applications and each has its own capabilities and drawbacks. If an incorrect model is chosen the quality and accuracy of the flow results will be degraded. Therefore, it is important to select a range of turbulence models that are assumed to be appropriate and determine a sensitivity analysis to conclude which is most suitable for the particular configuration (Shauin, Yayla, Canpolat \& Akilli, 2011). For the purpose of this work, simulations were carried out utilising the SAE, $\mathrm{k}-\omega$, LEA and SST turbulence models. While more powerful turbulence models were available, such as Detached Eddy Simulation and Large Eddy Simulation, these were not used due to complexity and related increased demand on computational power and time (The German Aerospace Center [DLR], 2010). These turbulence models were compared in detail using; force and moment graphs, surface contour plots and pressure coefficient graphs. The comprehensive study of these findings was made in Pevitt \& Alam (2011). These findings indicated that based on available computational power and feasible time constraints the most accurate models were developed when utilising the SAE turbulence model. Based on these studies, where possible the SAE turbulence model was used for the dynamic simulations that are to be made in this paper.

\subsection{Influence of Configuration Change}

When analysing the flow over the configuration it was noted that more than one layout for the model can be used. The two main alternatives looked at: a) the possibility of using a half model configuration, and b) effect of the wind tunnel sting attachment. The purpose of simulating a half model is to prove the results for the full and half model are the same. If this is true, then it is possible to make a flow analysis with greater mesh refinements over a half model (Stolz, 2006). It was also important to ensure that the sting attachment was not greatly affecting the wind tunnel results compared to the CFD simulations. Then determine if it was required to model this attachment 
to ensure an accurate representation of the results. In previous studies it has been suggested that the lack of a sting on the model can affect the pitching moment results (Frink, 2010).

Detailed analysis was completed previously both on full and half model simulations and with and without sting attachments. These simulations were run over a large range of AoA to gain a detailed overview of their effects. Based on these results it was seen that all of the features of the flow were the same for both the full and half model, allowing for further testing to be completed on half models (Pevitt \& Alam, 2011). When testing the effects of sting attachment it was seen that in general the sting caused the pitching moment results to translate upwards. As the results for pitching moment coefficients were generally under predicted, in previous finding, the upwards shift was a beneficial translation (Pevitt \& Alam, 2011).

\subsection{Influence of Discretisation Parameters}

Two of the discretisation parameters reviewed here are Preconditioning and the Dissipation, as these can greatly affect the simulation results. Preconditioning affects the assumptions made in the calculation process (Blazek, 2001). The simulations work through the use of the governing equations, assuming that the flow is incompressible. As the flow over the configuration is only 0.17 Mach the assumptions of incompressible flow is not completely valid (Eidgenössische Technishe Hochschule Zürich [ETH], 2010). The goal of preconditioning is to implement a correction factor to account for these assumptions and to improve the convergence of the numerical schemes at low Mach numbers. Based on this it is expected that its implementation should improve the results discussed previously (Lomax, Pulliam, \& Zingg, 1999).

The addition of preconditioning to the simulations was reviewed in detail (see Pevitt \& Alam, 2011). The addition of preconditioning has both positive and negative effects for all AoA when looking at the pitching moment coefficient values. It was noted that using preconditioning causes beneficial effects on the accuracy of the results, with a greater emphasis for lower AoA values. Though it was important to note that it also had negative effects for the lift and drag coefficient results, indicating its use would be highly dependent on the desired results.

Dissipation also effects the assumptions made in the calculation process. Dissipation refers to the degradation of the intensity in vortical flow (Blazek, 2001). The parameters associated with this value will affect how the turbulence model calculates the unsteady turbulent flow over the configuration. If the flow does not dissipate fast enough the results will indicate much large vortices over the aircraft than expected and there merging or separation will be delayed until higher angles of attack (Blazek, 2001). If the flow dissipates too fast then the vortices will disperse too early and the flow will merge and become separated at AoA much lower than what would be expected from experimental data (Celik, 2004). The Dissipation parameters are based on the 2nd order and 4th order dissipation coefficients.

A range of simulations was undertaken changing the 2nd and 4th order dissipation values to determine their separate and joint effects by Pevitt \& Alam (2011). Three different combinations were run at two separate AoA. These results were then represented with force and moment graphs. The results indicated that at low AoA, the changes in the pitching moment coefficients were negligible despite any changes in the dissipation values. When reviewing the results at higher AoA, a more noticeable effect was evident. With these inputs it was observed that the pitching moment values that were previously over predicting the experimental data were translated back down onto the experimental results, helping to improve the accuracy (Pevitt \& Alam, 2011).

\subsection{Finalised Static Simulation}

Based on previous studies, a final static flow simulation model was developed to form the basis for further dynamic testing. The final flow simulations were run on a half model with 22.5 million nodes. This model also incorporated the sting attachment and the preconditioning and dissipation values, previously seen to help improve the simulation accuracy (Pevitt \& Alam, 2011). This model implemented the lessons seen previously together to gain the most accurate results possible with the available resources. This model was then used as a reference guide for further testing where possible.

Detailed explanations of the complexities in the static flow over the delta wing were made by Pevitt \& Alam (2011). The Study indicated that the key flow characteristics over this model were difficult to determine and predict. This means it will be difficult to directly represent the dynamic data as well. Additionally studies to consider a more detailed look at the flow characteristics were made through the use of surface pressure plots and pressure taps along the wing of the model. (Schütte, Hummel, \& Hitzel, 2010; Pevitt \& Alam, 2011). These graphs were able to clearly represent the formation and dispersion of the vortices over the configuration. These finding also indicated that though the static model struggled to represent the flow characteristics at higher AoA, 
up until $17^{\circ}$ AoA, good confidence could be put in the simulation. Similar limitations were also expected in relation to the dynamics results.

\subsection{Pitching Oscillations}

As mentioned earlier the overall purpose of this paper was to determine the feasibility of modelling dynamic derivatives in CFD. The dynamic derivatives help to characterise the aircraft in respect to its stability and control properties (Yang X. L. et al., 2011). It is an essential part of the development cycle in flight physics. With modern aircraft it is often necessary to design unstable aircraft configurations, making the knowledge of the stability and control margins critical for the design of the flight control systems (Ismadi, 2011). For many different reasons it can be desirable to develop unstable aircraft such as reducing radar cross section or increased manoeuvrability. This concept of unstable design also applies to the configuration used in this work. In the scope of this paper the goal was to be able to model the dynamic pitching coefficient of the aircraft under amplitude changes of $\pm 5^{\circ}$ with a pitching frequency of both $1 \mathrm{~Hz}$ and $3 \mathrm{~Hz}$.

The initial computational models were performed based on the statically determined model (see Pevitt \& Alam, 2011). A range of simulations were performed by changing different parameters of a static model to firstly try and gain the optimal static design. Initial dynamic tests were performed based on the initial static results. These static results were based on a model with a mesh refinement of 10.5 million nodes and were run using a SAE turbulence model. These initial static results can be seen in Figures 6 to 8 compared to the experimental dynamic wind tunnel results. The reason this model was used first, rather than a more refined model, is that the dynamic tests were run in parallel to the static ones. This means that when the computational simulations began, the findings of a more refined model had not yet been made. The effect of the more refined model is discussed later.

The first step in the calculation of dynamic pitch oscillations is to find the moment and forces associated with each run. This is done based on the static solution found from the 10.5 million node models. The solution outputs for $5^{\circ}, 10^{\circ}, 15^{\circ}$ and $25^{\circ}$ were taken. Then each of these models was re-run under dynamic pitching oscillations. Each model was run with amplitude of $\pm 5^{\circ}$ with the first computation being done at $1 \mathrm{~Hz}$. This was chosen for initial testing as in previous studies it was noted that the slower frequencies were harder to model accurately (Zhang, 2011). The reason that slower frequencies are harder to model is that the lower frequencies undergo more non-linear behaviour (Liu \& Chen, 2011). This is due to the flow dynamics having enough time to transition between states at lower frequencies. For higher frequencies the flow will not have enough time to transition and will remain separated once it is disturbed, this causes an overall more linear behaviour (Rohlf, 2010).

The dynamic simulations were run using the same parameter settings as the static runs found by Pevitt $\&$ Alam (2011). Each cycle was run with 100 iterations and 100 inner iterations. Each model was run for a total of 3 cycles to ensure the results were well converged. In order to run the simulations at $1 \mathrm{~Hz}$ the TAU inputs corresponded to a reduced frequency of rotation, 0.06 . These simulations were run and provided dynamic force and moment graphs. These results are illustrated in Figures 6 to 8 . The equivalent experimental data that corresponds with these values is also shown in Figures 3 to 5.

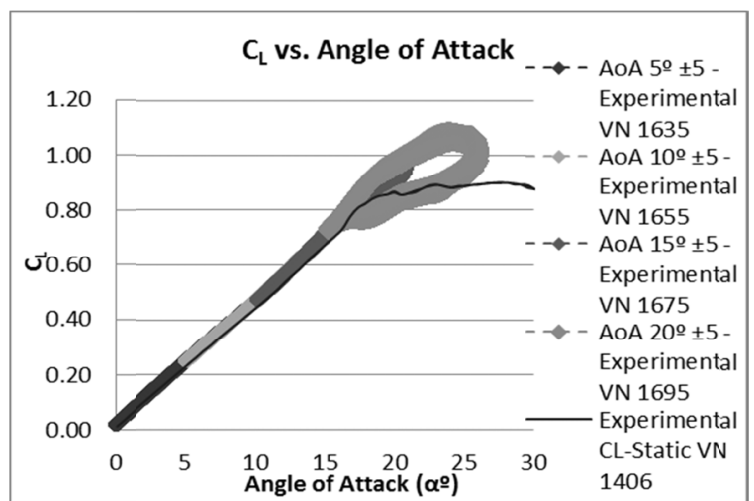

Figure 3. Experimental Data - CL vs. Angle of Attack

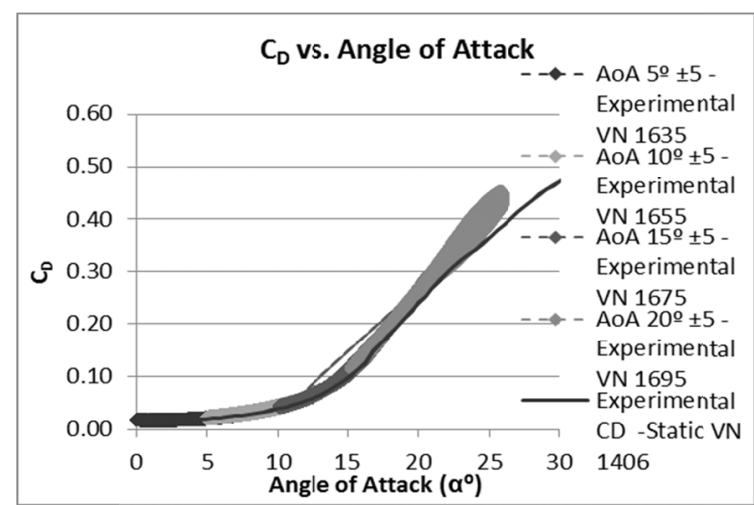

Figure 4. Experimental Data - C vs. Angle of Attack 


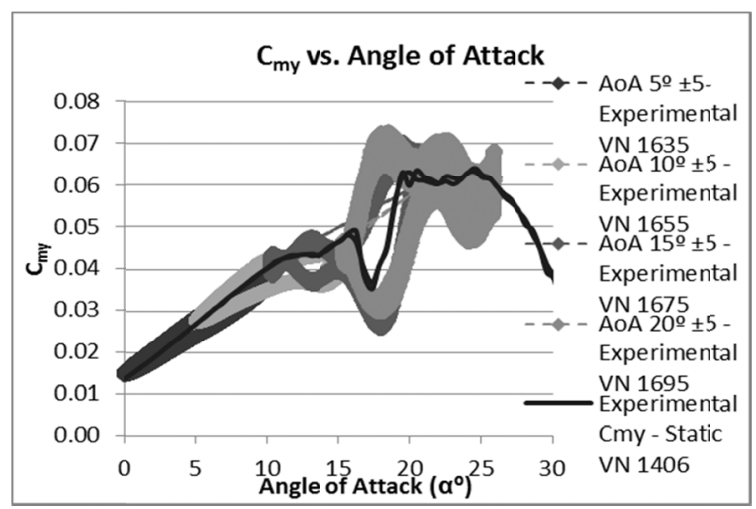

Figure 5. Experimental Data - Cmy vs. Angle of Attack

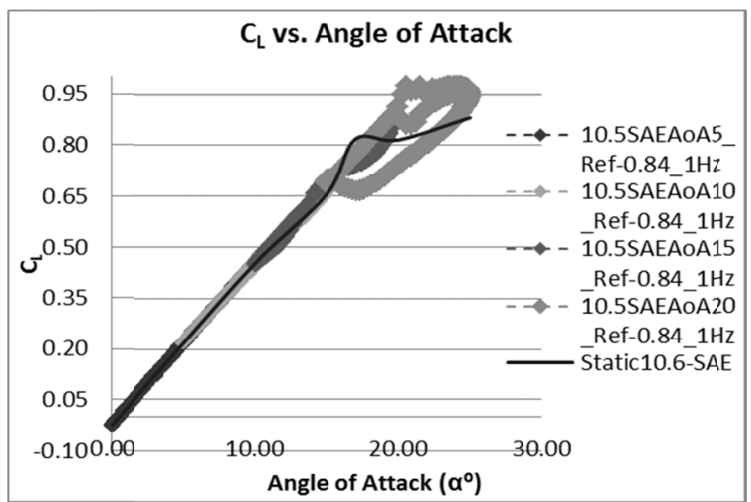

Figure 6. Dynamic Tests, for $5^{\circ}, 10^{\circ}, 15^{\circ}$ and $20^{\circ}$ with $\pm 5^{\circ}, \mathrm{CL}$ vs. Angle of Attack

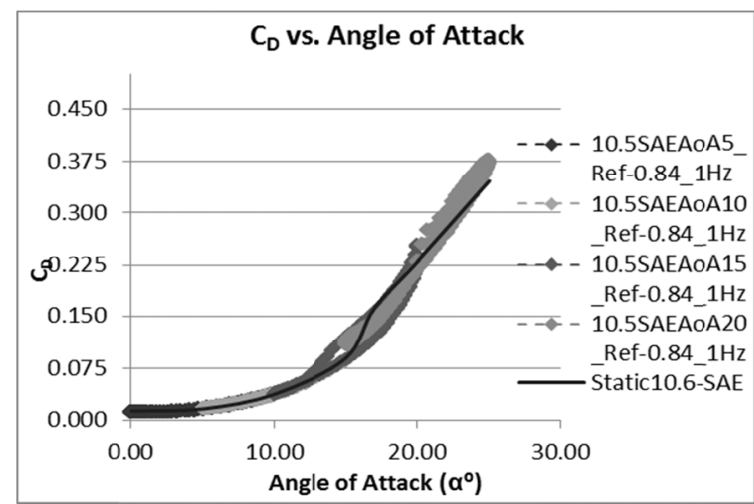

Figure 7. Dynamic Tests, for $5^{\circ}, 10^{\circ}, 15^{\circ}$ and $20^{\circ}$ with $\pm 5^{\circ}, \mathrm{CD}$ vs. Angle of Attack

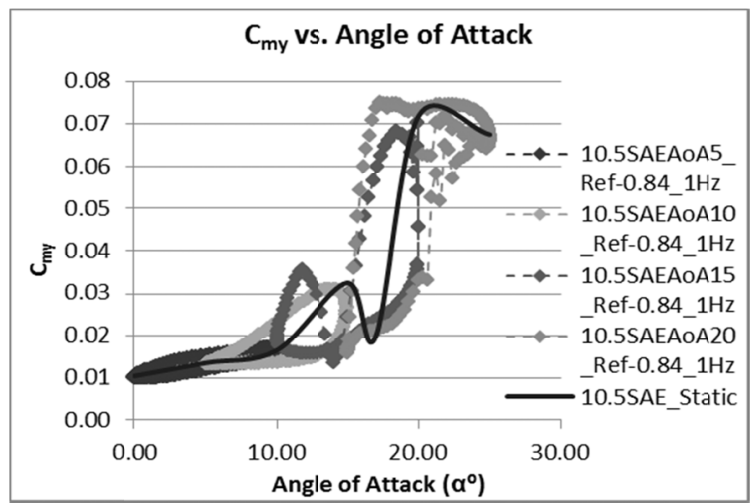

Figure 8. Dynamic Tests, for $5^{\circ}, 10^{\circ}, 15^{\circ}$ and $20^{\circ}$ with $\pm 5^{\circ}$, Cmy vs. Angle of Attack

As observed in the experimental data, the dynamic simulations follow the trend provided by the static curve. This is important to note as it can clearly be seen that if there are deviations in the static results compared to experimental values, then it not possible to expect the dynamic solutions to be completely accurate. This means, the first step in ensuring an accurate dynamic solution would be to define an accurate static solution. Without this reference to begin the dynamic solutions from, ideal dynamic modelling cannot be attempted. If the dynamic solutions can be accurately modelled around the static solution, then the concept and feasibility of the dynamic modelling can be useful.

Figures 6 to 8 clearly indicate that the most complex and least linear results are seen in the pitching moment characteristics. Compared to the experimental values, there is the least amount of agreements in the results for the pitching moment coefficients. As a result the pitching moment results will be focused on for further modelling and analysis. It can be noted that the improvement in pitching moment results will coincide with 
improvements in coefficients of lift and drag results (Kwak, 2010). A detailed comparison of pitching moment results with experimental results are shown in Figure 9.

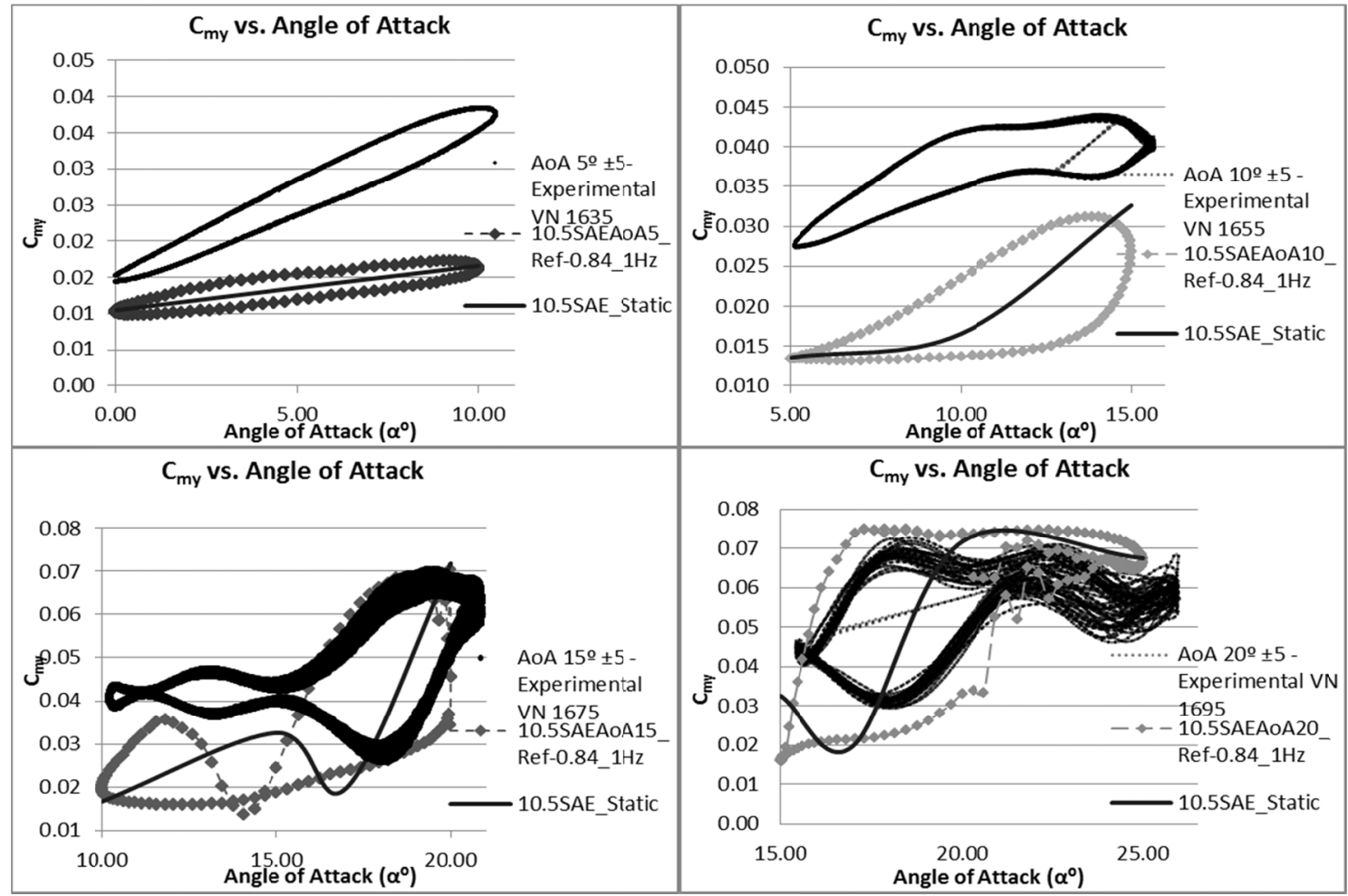

Figure 9. Experimental Data vs. Dynamic Tests, for $5^{\circ}, 10^{\circ}, 15^{\circ}$ and $20^{\circ}$ with $\pm 5^{\circ}$

When reviewing the dynamic results for the pitching moment, it is observed that there are large deviations in the results. This was expected, as the static results had such large deviations compared to the experimental results. It has been noted that the dynamic results follow the static results all the time. A therefor high level of accuracy was not expected for the dynamic $C_{m y}$ values. When reviewing the $C_{L}$ and $C_{D}$ dynamic graph it was seen that for all AoA, even where the results deviated from the experimental ones, the flow phenomena was still captured relatively well. The simulation data still showed the same shape and characteristics as the experimental data. When reviewing the dynamic $\mathrm{C}_{\mathrm{my}}$ values it was seen that the values were not only translated away from the experimental results but also the flow shape and characteristics were not captured.

At $10^{\circ}$ with an oscillation of $\pm 5^{\circ}$, for the dynamic pitching moment, it is noted that the results are not only translated down but also not representative of the experimental data at all. This is the region of AoA where the wing tip vortex is beginning to move inboard and start to form with the thickness vortex (Shauin, 2011). This phenomenon was very hard to capture accurately in the static model, so it is no surprise that it has not been captured exactly for the dynamic model. From the static results seen in Pevitt \& Alam (2011), it was observed that the flow characteristics could be captured at $15^{\circ}$ and $17^{\circ}$, though the way the vortices move in-between these AoA is unknown, it may be this change in transition that is not being captured correctly by this model. It would be expected that the addition of mesh refinement in these areas would help to better capture this region more accurately as well as the utilisation for a more accurate turbulence model (Blazek, 2001).

Looking at other AoA results for the dynamic pitching moment, it is noted that only some parts of the curve are in good agreement with the experimental results. For the lower AoA around $10^{\circ}$ to $17^{\circ}$ the flow is much more complicated. It is this region of the curve not only struggles to provide the correct data but also fails to represent the same characteristics of the flow. The cause of this is again due to the inability to model the transition of the tip vortex as it merges into the thickness vortex, further inboard of the leading edge (Liu \& Chen, 2011). Once the AoA increases more and the vortices have merge with the apex vortex, causing flow separations, the simulation is able to capture the flow more accurately again (Cummings, 2010). This is why between $17^{\circ}$ and $20^{\circ}$ the graph is translated back towards the experimental data and captures characteristics of the flow quite well.

As the flow beyond $17^{\circ}$ has now become detached, as a result of the apex, thickness, and tip vortex all merging. 
This actually makes the flow much easier to simulate, as it is fully separated (Vicroy, 2010). This means at $20^{\circ}$ dynamic pitching moment it can be seen that the results are in much more agreement. There are still some variances in the result, though this is also noted in the baseline static results. Unlike the lower AoA results, the characteristics of the flow for higher AoA appear to be modeled reasonably well. Additionally, with all of the experimental dynamic data, especially at $20^{\circ}$, there are large deviations in wind tunnel results. This implies that this flow is complicated and hard to capture and also that the flow is not necessarily easily repeatable. This makes it harder to be captured accurately by CFD. Therefore it can be stated that for the $20^{\circ}$ dynamic pitching moment graph, the simulation is in reasonably good agreement with the experimental data.

Based on the simulations shown in Figure 9 it was again clearly evident that the dynamic model would follow the static model results. Therefore an improved static model would lead to the $\mathrm{C}_{\mathrm{my}}$ values closer matching the correct characteristics at low angles of attack, and provide reasonably good agreement at high angles of attack. Though between AoA of $10^{\circ}$ and $17^{\circ}$ the flow becomes very complicated and the dynamic graphs struggle to accurately represent the characteristics of the flow. It is expected that with further mesh refinements this would be improved (Stolz, 2006).

Based on the results the dynamic data with a more refined model was selected. The 22.5 million node model was found to be a beneficial choice for the simulations. Additionally the advantages of sting attachment, dissipation and preconditioning use found by Pevitt \& Alam (2011) were utilised. Due to this, two new tests were run. One was based on the 22.5 million nodes, half model with sting, and the other was based on the same model with both dissipation and preconditioning parameters altered to better capture the flow. The goal from this was to try and match the testing performed on the static results to the capabilities of the dynamic results. Despite several attempts, a simulation could not be made that would allow for the solution to converge when both the preconditioning and dissipation parameters were in place. These models would results in failure due to numerical errors. Tests were then performed on both preconditioning and dissipation separately. It was then found that the dissipation input was leading to numerical errors.

When the preconditioning was used alone, it was found that the model would converge, only when refinements were made to the inner time steps in the solver. To try and help the process the model was rerun with precondition and the inner time steps were increased from 200 to 400 (Blazek, 2001). This improved the convergence greatly but still did not fully converge the results. Due to this, the preconditioning was not used for further studies, as further time step refinements would increase the computational time beyond the feasible limits for this study. To indicate the quality of the standard 22.5 million node model and the benefits of precondition the $\mathrm{C}_{\mathrm{my}}$ graphs at $20^{\circ}$ with and without preconditioning are shown in Figure 10.

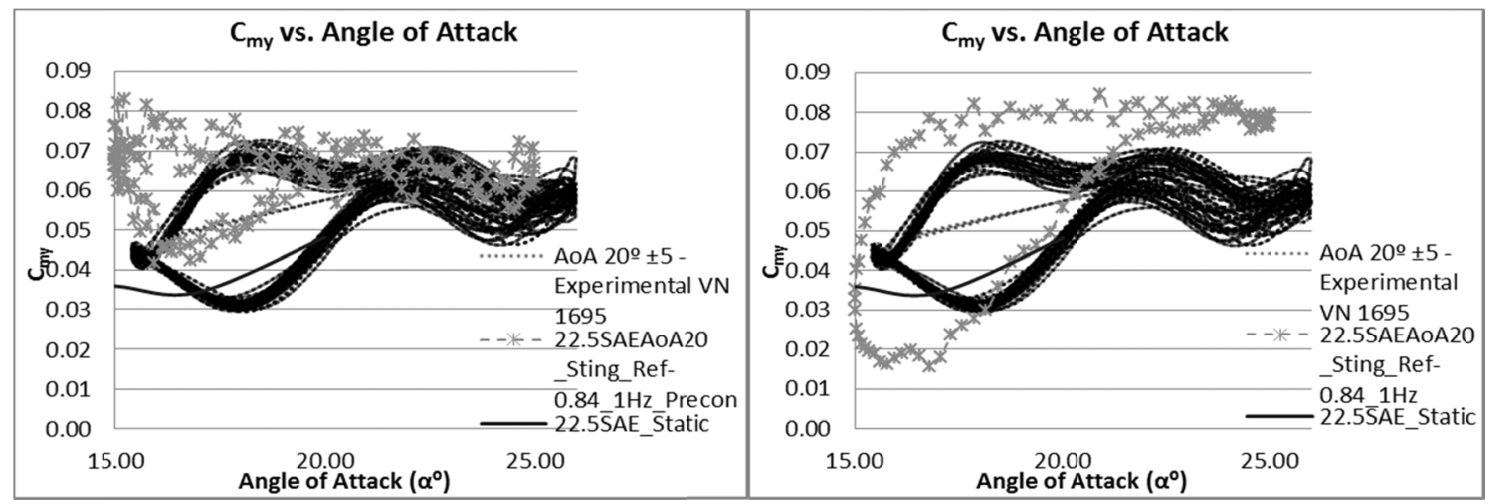

Figure 10. Experimental Data vs. Dynamic Tests, at $20^{\circ}$ for Standard 22.5 million nodes (left) preconditioning (right)

In Figure 10, it was observed that the additional mesh refinement changed the shape of the $\mathrm{C}_{\mathrm{my}}$ dynamic graph but did not improve its overall accuracy. Due to this, a detailed decision of its benefits could not be made without further testing of additional AoA. When looking at the preconditioning model it appears that the results were not only improved greatly but also would follow the characteristics of the flow much better. As these results would not fully converge it was not possible to use them in good confidence thereafter. It is expected that with additional time steps this model would converge further and would result in the more accurate and viable results (ETH, 2010). Although with the available computational power and time frame it was not possible to further refine the time steps on this model to determine the possible accuracy achievable. Due to this convergence problem, further testing was done on the 22.5 million nodes, half model with sting without preconditioning. 
Additional tests were performed at $10^{\circ}$ and $15^{\circ}$. The results are shown in Figure 11.
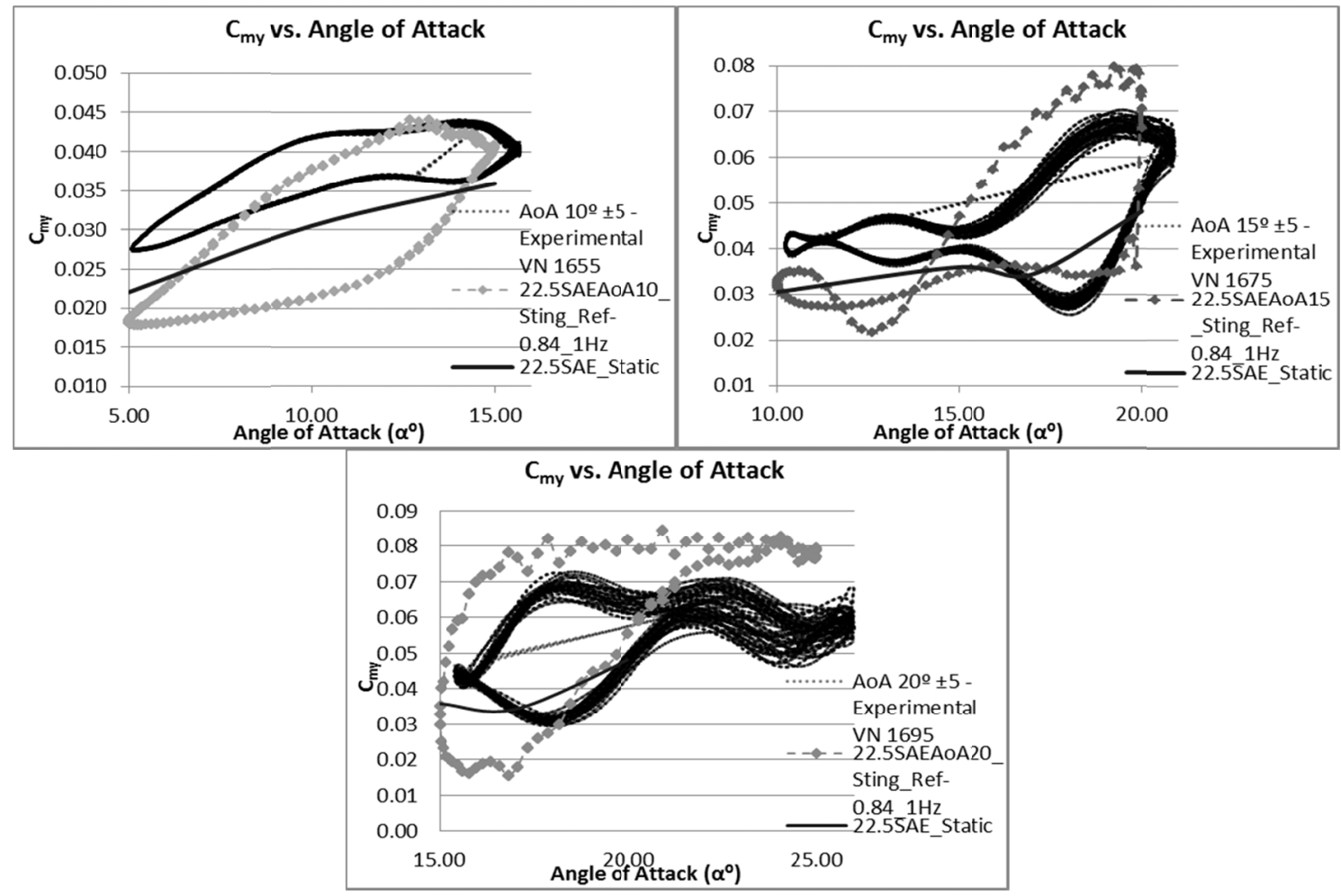

Figure 11. Experimental Data vs. Dynamic Tests - Cmy, for $10^{\circ}, 15^{\circ}$ and $20^{\circ}$ with $\pm 5^{\circ}$

The effect of the 22.5 million nodes mesh is more noticeable for the pitching moment graphs in Figure 11. When looking at the solution at $10^{\circ}$ it is noted that due to the improvement in the static results, the dynamic results have translated up noticeably and are now much closer to the experimental data. The characteristics of the flow have also improved, though as this is an area of the complicated flow, the characteristics indicated are still very different to the experimental data. It is expected that being able to get the preconditioning and dissipation parameters to work would allow for this characterisation of the flow to be modelled in a greater detail (Lomax, 1999).

For the Dynamic $\mathrm{C}_{\mathrm{my}}$ values at $15^{\circ}$ degrees it is noted that the improvement in static results have caused the dynamic results to translate upwards, improving the quality of the results. Additionally the graph now represents the flow characteristics better. However on the downwards stroke (or the top line) the results dip and fall below the upward stroke line. This characteristic is not present in the experimental data. As mentioned earlier, the 22.5 million node model begins to merge its leading edge and thickness vortices sooner. This effect is due to the dip in the $\mathrm{C}_{\mathrm{my}}$ results. It was observed that for the static results, this improved when preconditioning and dissipation parameters were added to the solution (Pevitt \& Alam, 2011). Therefore, it is believed that if the dynamic simulation is run with these parameters, the dip in the results would be attended to and the characteristics of the flow will be more accurate.

For the $20^{\circ}$ Dynamic $\mathrm{C}_{\mathrm{my}}$ results, it was noted that the solution was again translated up as a result of the new static solution. Overall, the accuracy and characteristics of the flow in this region do not vary notably. The results on the lower AoA side around $15^{\circ}$ have become closer to the experimental results due to the upwards translation of the graph. At the same time, the upwards translation has caused the results around $25^{\circ}$ to move away from the experimental results slightly. In both the 10.5 million node model and the 22.5 million node model the characteristics of the flow do not change significantly. Based on the one $1 \mathrm{~Hz}$ results it was found that the 22.5 million node model did provide the most accurate results but was still lacking as it did not fully characterise the dynamic flow across all AoA. It is believed that with the addition of the dissipation and preconditioning parameters, the quality of the results would noticeably improve.

In addition to these tests, some initial testing was performed on models pitching at $3 \mathrm{~Hz}$ oscillation. These models were also run in unison to both sets of testing. Due to this and because of a lack of time available the 3 
$\mathrm{Hz}$ model testing was performed on the 9.6 million node model with a $\mathrm{k}-\omega$ turbulence model. This model was found to be less accurate than the 22.5 million node SAE model based on the static results (Pevitt \& Alam, 2011). Despite less accuracy and due to time and resource limitations the force and moment results for the $3 \mathrm{~Hz}$ testing were performed for $10^{\circ}$ and $20^{\circ}$ AoA using the 9.6 million node model. By placing the model in a $3 \mathrm{~Hz}$ oscillation the reduced frequency of rotation was 0.196 . The pitching moment results are illustrated in Figure 12. Despite the $3 \mathrm{~Hz}$ model being based on a much less accurate reference model, Figure 12 illustrates that the majority of the results were quite similar to the experimental data and showed good agreement with flow characteristics.

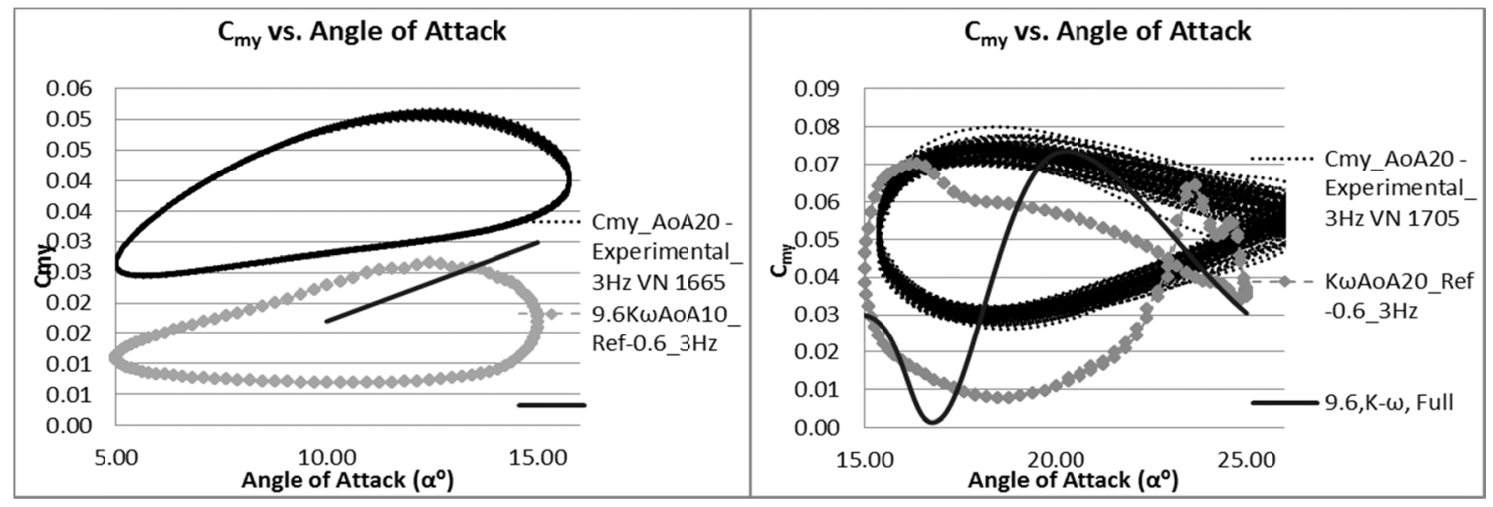

Figure 12. Experimental Data vs. Dynamic Tests at $3 \mathrm{~Hz}$, for $10^{\circ}$ and $20^{\circ}$ with $\pm 5^{\circ}$

After reviewing the $\mathrm{C}_{\mathrm{my}}$ dynamic data, it was observed that at $10^{\circ}$ the $3 \mathrm{~Hz}$ still struggles to model the results accurately. It appears that the $10^{\circ} \mathrm{C}_{\mathrm{my}}$ graph is the hardest to model for both $1 \mathrm{~Hz}$ and $3 \mathrm{~Hz}$. For the $3 \mathrm{~Hz}$ graph the flow characteristics and values are much closer than what was found in the $1 \mathrm{~Hz}$ graph. Overall the results are still quite different compared to the experimental data for $3 \mathrm{~Hz}$. It is expected that if the more refined mesh with the SAE model, preconditioning and dissipation were used for the $3 \mathrm{~Hz}$ testing the results would be closer.

For the $20^{\circ}$ results, the simulation data is quite close to the experimental data. The graph appears to represent the flow characteristics accurately, but is translated down slightly. It is known that this downwards translation is due to the less accurate static data from the k- $\omega$ model. Therefore it is believed that these results can be further improved through the use of the more accurate base model. Overall, it was found that the $3 \mathrm{~Hz}$ data was much easier to model than the $1 \mathrm{~Hz}$ data. Even using a much less accurate static model the results were both quite accurate and indicated a good characterisation of the flow. Based on the results, it is believed that with the use of the 22.5 million node model with the SAE turbulence model, preconditioning and dissipation the results would be much more accurate.

\section{Conclusions}

Based on the findings from CFD simulations, a final simulation model was created utilising a range of beneficial simulation parameters. The final model was based on 22.5 million node half model, with the sting attachment, preconditioning and dissipation parameters.

The findings from this study indicate that it is vital to ensure an adequate mesh refinement with a definite focus on leading edge refinements. Otherwise, the results can deviate largely from the experimental findings. The addition of the sting, preconditioning parameters and increased dissipation parameters can lead to more accurately refined models, except for the $17^{\circ} \mathrm{AoA}$.

The study indicates that with the appropriate mesh refinement and flow parameters, the TAU code is capable of representing the force and moment coefficient results to an appropriate level of accuracy at lower AoA. However, in order to truly represent the overall flight envelope additional more specialised meshes and further refinements are required. This can be the concept development of two separate models, one for lower AoA simulations and the other is for higher AoA simulations.

For the dynamic results, studies were completed at $1 \mathrm{~Hz}$ and were based on the original 10.5 million nodes, SAE model and at $3 \mathrm{~Hz}$ on the 9.6 million nodes $\mathrm{k}-\omega$ model. Initial findings indicated that the dynamic results in all force and moment graphs were directly related to the static results of the same model. Therefore high levels of accuracy were not possible unless the static models were improved. Despite their variances in numerical values, the $C_{L}$ and $C_{D}$ results were capable of accurately indicating the flow characteristics, while the $C_{m y}$ results 
appeared to vary both in numerical results and flow characteristics, especially in the region of flow separation.

The numerical results and the flow characteristics improved for the mesh refined models. These improvements indicated notable beneficial changes in the flow characteristics at all AoA used in this study.

Based on the current accuracy of the dynamic models, the additional model refinement is needed. As the dynamic results follow the static findings, improvements made in the static results would greatly improve the dynamic results. It can be suggested that at current accuracy levels, it was not be possible to model the $1 \mathrm{~Hz}$ data in good confidence. However, the $3 \mathrm{~Hz}$ results can be modelled in quite good confidence, despite slight deviances were found in the $\mathrm{C}_{\mathrm{my}}$ results at $10^{\circ}$ to $17^{\circ} \mathrm{AoA}$. It is also believed that with further computational simulations with a more accurate model further improvement can be achieved in high frequency dynamic modelling.

\section{Acknowledgements}

The authors are highly grateful to Dr. Stephan Hitzel and Dr. Herbert Rieger, from CASSIDIAN - Air systems for their assistance and support with the organisation and preparation of this work, as well as for their time and effort spent in ensuring it was produced to a high standard.

\section{References}

Anderson, J. D. (2007). Fundamentals of Aerodynamics (4th ed.). New York: McGraw-Hill.

Blazek, J. (2001). Computational Fluid Dynamics: Principles and Applications (1st ed.). Kidlington, Oxford: Elsevier Science Ltd.

Celik, I. B. (2004). Procedure for Estimation and Reporting of Discretization Error in CFD Applications. Mechanical and Aerospace Engineering Department, West Virginia University, USA.

Century of Flight, Development of Aviation Technology - Delta Wings. (2010). Retrieved from http://www.century-of-flight.net/ [January 2011]

Cummings, R. M., Jirásek, A., Petterson, K., \& Schmidt, S. (2010). SACCON Static and Dynamic Motion Flow Physics Simulation Using Cobalt. AIAA, 4691.

The German Aerospace Center (DLR) - Institute of Aerodynamics and Flow Technology. (2010). Technical Report - Technical Documentation of the DLR TAU-Code, version: 2010.1.0.

Eidgenössische Technishe Hochschule Zürich (ETH) (2010). Governing equations of fluid dynamics. Retrieved from http://www.ltnt.ethz.ch/teaching/IntrotoCFD/cfd_class_march_2_2010.pdf

Frink, N. T. (2010). Strategy for Dynamic CFD Simulations on SACCON Configuration. AIAA, 4559.

Gursul, I., Gordinier, R., \& Visbal, M. (2005). Unsteady Aerodynamics of Nonslender Delta Wings., s.l.: Elsevier Ltd.

Gursul, I. (2004). Recent Developments in Delta Wing Aerodynmaics. The Aeronatical Journal, September.

Hübner, A. R. (2007). Experimental and Numerical Investigations of Unsteady Aerodynamic Derivatives for Transport Aircraft Configurations. AIAA, 1076.

Ismadi, M. Z. (2011). Experimental control of vortex breakdown by density effects. Physics of Fluids.

Kollmann, W. (2011). Accurate numerical simulations of vortex flows. I: Vortex breakdown. Computers and Fluids, 103-109. http://dx.doi.org/10.1016/j.compfluid.2010.09.018

Kuo, K. T., Liu, W. H., Hsieh, S. J., \& Chou, J. H. (1995). Flow Developments Above 50-Deg Sweep Delta Wings with Different Leading-Edge Profiles. Journal of Aircraft.

Kwak, H. Y. (2010). Vortical flow control over delta wings with different sweep back angles using DBD plasma actuators. 5th Flow Control Conference, AIAA-2010-4837, Chicago, USA.

Liu, C., \& Chen, L. (2011). Parallel DNS for vortex structure of late stages of flow transition. Computers and Fluids, 129-137. http://dx.doi.org/10.1016/j.compfluid.2010.11.006

Lomax, H., Pulliam, T. H., \& Zingg, D. W. (1999). Fundamentals of Computational Fluid Dynamics. NASA Ames Research Center \& University of Toronto Institute of Aerospace Studies.

Pevitt, C., \& Alam, F. (2011). Feasibility of Static Computational Fluid Dynamics Simulations around a Specialized Delta Wing Configuration. 1st International Conference on Mechanical Engineering and Renewable Energy, ICMERE, Venue: CUET, Chittagong, Bangladesh. 
Schütte, A., Hummel, D., \& Hitzel, S. M. (2010). Numerical and experimental analyses of the vortical flow aroundthe SACCON configuration. AIAA, 4690.

Shauin, B., Yayla, S., Canpolat, C., \& Akilli, H. (2011). Flow Structure Over the Yawed Nonslender Diamond Wing. Aerospace Science and Technology. http://dx.doi.org/10.1016/j.ast.2011.06.008

Stolz, S. (2006). Advanced CFD Methods. Eidgenössische Technishe Hochschule Zürich (ETH), Lecture Notes.

Surrung. (2009). Pakistan Defence-Wing Planforms. Retrieved from http://www.defence.pk/forums/india-defence/4346-lca-news-discussions-316.html

Vicroy, D. D., Loeser, T. D., \& Schütte, A. (2010). SACCON Forced Oscillation Tests at DNW-NWB and NASALangley 14X22-foot Tunnel. AIAA, 4394.

Visbal, M. R. (1995). Computational and Physical Aspects of Vortex Breakdown on Delta Wings. 33rd AIAA Aerospace Sciences Meeting and Exihibit, Reno, NV, USA.

Yang, X. L., Liu, W., Wang, H. B., \& Zhao, Y. F. (2011). Numerical investigation of a slender delta wing in combined force-pitch and free-roll. Proceedings of World Academy of Science, Engineering and Technology, 895-901.

Zhang, M. (2011). Unsteady characteristics of breakdown vortices over delta wing. Applied Mechanics and Materials, 66-68, 1874-1877. http://www.scientific.net/AMM.66-68.1874 